\title{
Tratamiento quirúrgico de las fracturas de
}

\section{metacarpianos y falanges}

\author{
R. Cancelo Barea \\ Práctica Privada. SeVIlla \\ Correspondencia: \\ consulta@ramoncancelo.com
}

\section{INTRODUCCIÓN}

T as fracturas de los metacarpianos y de las falanges de las manos son las más frecuentes de la extremidad superior, llegando a alcanzar el $10 \%$ del total de las fracturas. Las de la falange distal de los dedos son las más frecuentes del esqueleto, seguidas de las de la falange proximal, siendo los dedos más afectados el $1^{\circ}$ y el $5^{\circ}$, y su frecuencia es mayor en varones, sobre todo entre los 10 y 40 años.

Siempre han existido con respecto a estas fracturas una serie de tópicos, como el de ser consideradas lesiones banales, que la mayoría son funcionalmente estables desde el principio y por tanto hay que tratarlas de forma conservadora, y que suelen evolucionar bien. Si bien esto es cierto en parte, solo es aplicable a fracturas cerradas no desplazadas o con cierto desplazamiento, pero estables tras la reducción. Sin embargo, hoy día, y sobre todo en el ámbito laboral y deportivo, estamos viendo lesiones que no cumplen estos criterios y en las que por tanto es preferible realizar un tratamiento quirúrgico que estabilice y fije la fractura de forma tal que se pueda comenzar lo antes posible el tratamiento rehabilitador.

Ya dijo el Dr. Alfred Swanson ${ }^{1}$ que las fracturas de la mano pueden complicarse con deformidades por falta de tratamiento, rigidez por tratamiento excesivo, y deformidades óseas y rigidez por tratamiento incorrecto.

A nuestro criterio la popularidad del tratamiento quirúrgico en los últimos años ha venido dada por el progreso de los materiales, el diseño de los implantes e instrumental, las mayores ex- pectativas de la opinión pública, los avances en el diagnóstico por la imagen, la disponibilidad de especialistas en cirugía de la mano, los avances de la anestesia, y la mejora de la fisioterapia.

El objetivo principal del tratamiento siempre debe ser la recuperación rápida y completa de la función de la mano, y para conseguirlo debemos utilizar el método que creamos más adecuado en cada momento, quirúrgico o no, pero siempre con sentido común, ya que intentos muy agresivos de fijación interna pueden ocasionar lesiones de partes blandas, adherencias tendinosas, infecciones y reintervenciones. La fijación operatoria debe usarse de forma juiciosa y con la esperanza que el resultado definitivo sea tan bueno o mejor que el del tratamiento conservador.

Las principales indicaciones del tratamiento quirúrgico según $\mathrm{Stern}^{2}$ son las siguientes:

- Fracturas irreductibles.

- Malrotación.

- Fracturas intraarticulares.

- Fracturas subcapitales (falanges).

- Fracturas abiertas.

- Pérdida segmentaria de hueso.

- Politraumatismos con fracturas de las manos.

- Fracturas múltiples de la mano o la muñeca.

- Fracturas con lesiones de partes blandas (vasos, tendones, nervios, piel).

- Reconstrucción (por ej. osteotomía).

En la figura 1 podemos apreciar una fractura diafisaria del $5^{\circ}$ metacarpiano con ligero desplazamiento pero clara rotación, que hubiese dejado 


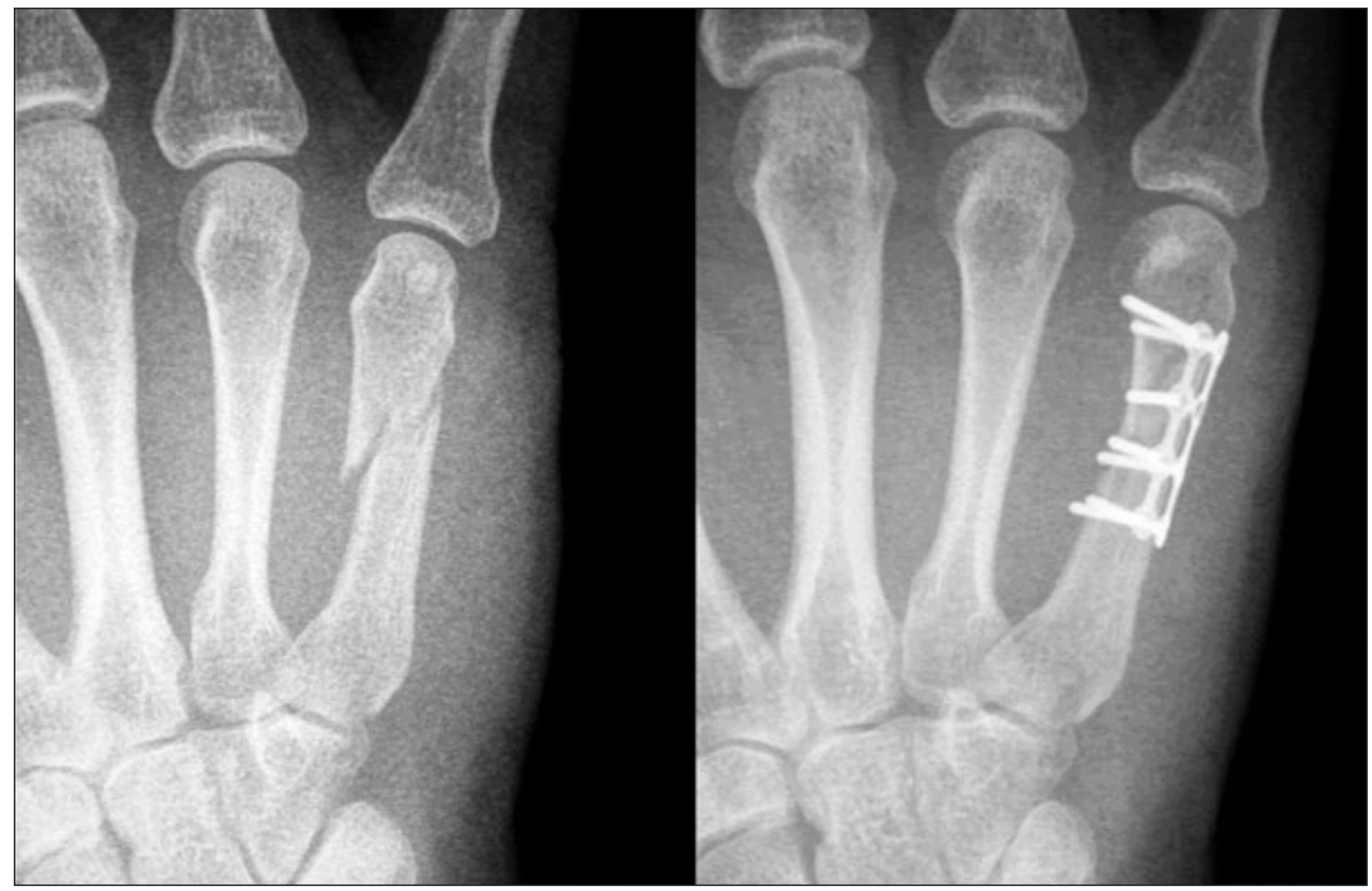

Figura 1: Fractura de metacarpiano malrotada.

secuelas si no se reduce correctamente. En este caso la fijación se realizó con miniplaca de titanio atornillada, para que el montaje fuese lo suficientemente estable que permitiese una movilización casi inmediata.

En la figura 2 apreciamos fractura de falange proximal con desplazamiento y acortamiento, que es tratada igualmente con síntesis con miniplaca atornillada.

En la figura 3 apreciamos fractura articular de base de falange proximal con hundimiento articular central y malrotación, que tras cirugía se consigue una reducción perfecta y estable.

En las fracturas-luxaciones de la base de los metacarpianos, sobre todo del segmento móvil $\left(4^{\circ}\right.$ y $\left.5^{\circ}\right)$, puede ser suficiente la reducción y fijación con agujas percutáneas transmetacarpianas o transarticulares, que se dejan hasta la consolidación de la fractura (Figura 4).

Especial mención requieren las fracturas de la base del pulgar, que se pueden clasificar en extra e intraarticulares.

Las extraarticulares o epibasales ${ }^{3,4}$ son fracturas frecuentes, generalmente de trazo transversal o ligeramente oblicuo y angulación de vértice

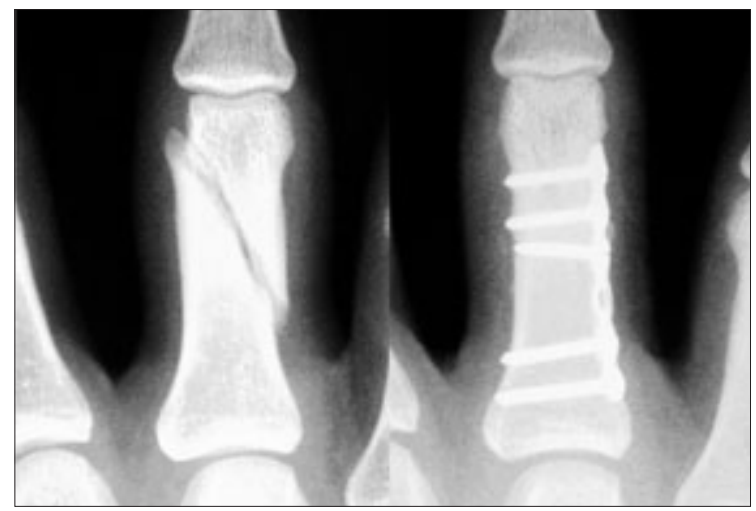

Figura 2: Fractura de falange proximal desplazada con acortamiento.

dorsal; el fragmento distal suele estar en aducción y flexión. Su tratamiento es no quirúrgico, ya que la reducción es generalmente estable, dejando el tratamiento quirúrgico con agujas de Kirschner, o rara vez con placas y fijadores para las inestables o con angulaciones mayores de $30^{\circ}$, con objeto de evitar la hiperextensión compensadora de la articulación metacarpofalángica del pulgar.

Las intraarticulares son la fractura de Bennett y la fractura de Rolando. 


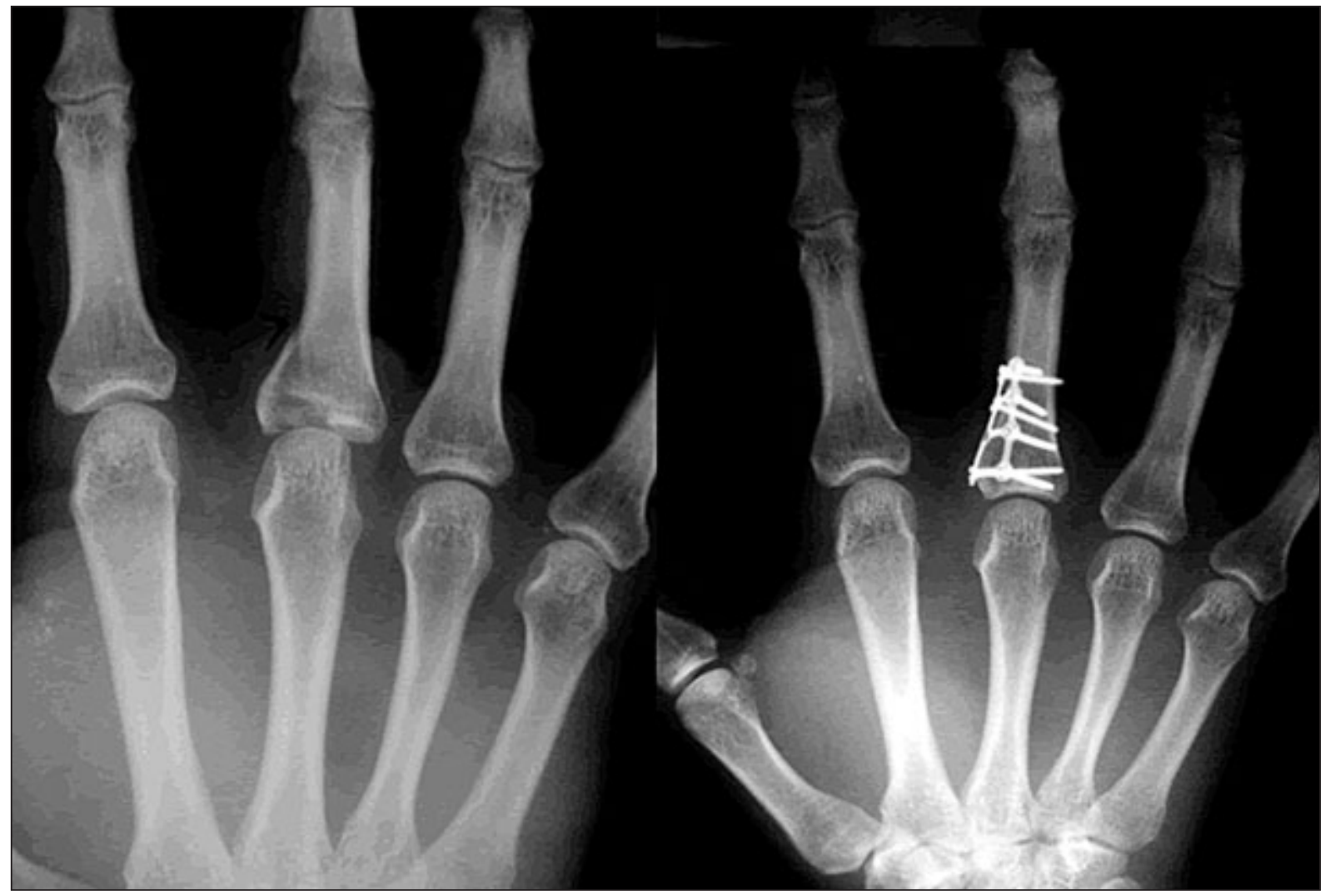

Figura 3: Fractura articular de base de falange proximal.

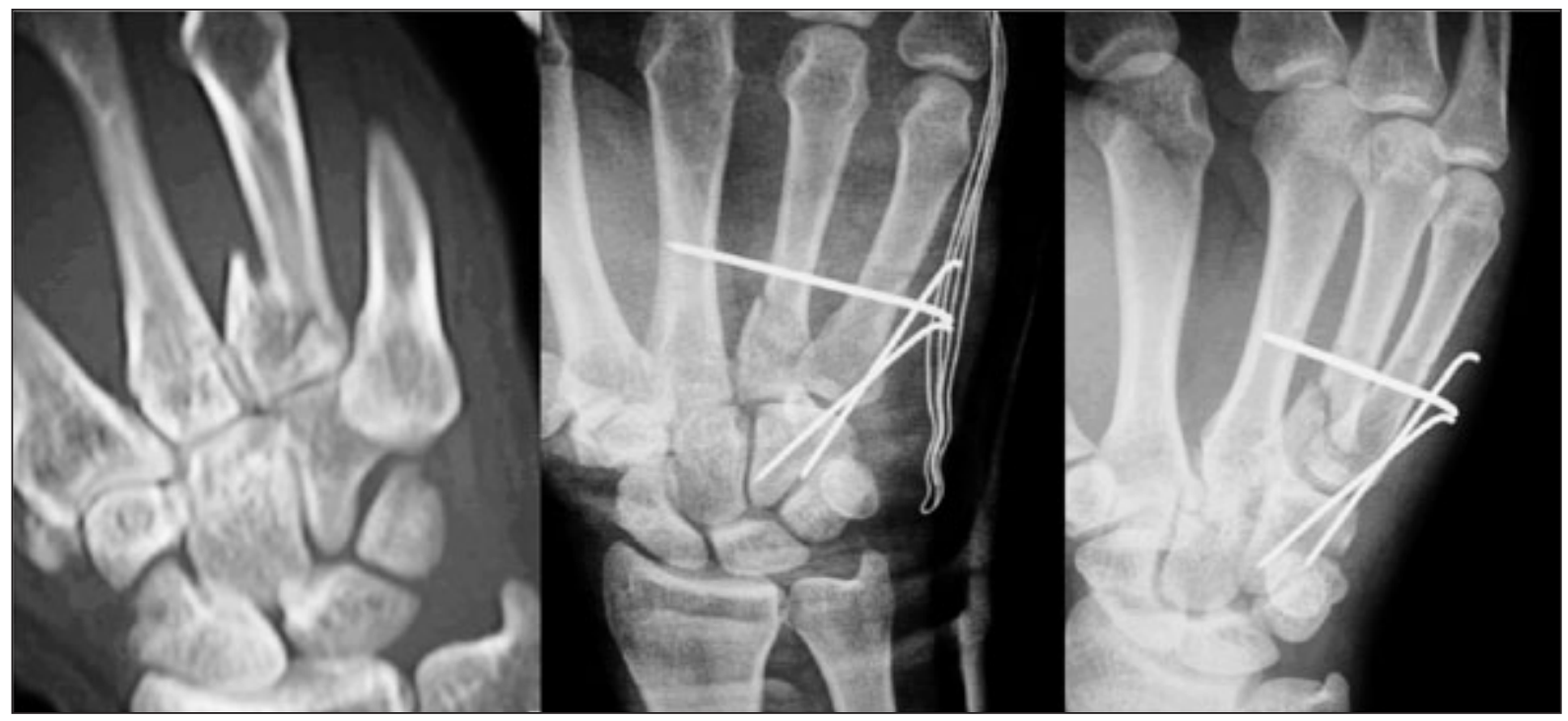

Figura 4: Fractura-luxación base metacarpiano. Fijación con agujas.

Edward Hallaran Bennett (1837-1907), cirujano Irlandés que describió la lesión que lleva su nombre en el año $1882^{5}$, tratándose de una fractura intraarticular de la base del $1^{\text {er }}$ metacarpiano, que comprende un fragmento óseo de tamaño variable y de forma piramidal, localizado en la zona volar y cubital, y que se mantiene unido al trapecio por el potente ligamento oblicuo anterior, mientras que el resto se desplaza hacia dorsal y radial por la tracción del abductor pollicis longus. Suele afectar con mayor frecuencia al sexo masculino y es más frecuente en caídas de bicicleta, moto y esquí. 
Para su diagnóstico es fundamental realizar bien la proyección radiológica, practicando una verdadera proyección lateral de la articulación trapeciometacarpiana. El tratamiento será no quirúrgico siempre que sea posible la reducción y posterior inmovilización, y quirúrgico cuando exista desplazamiento no reductible, escalón mayor de 1-2 mm entre los fragmentos, o que el tamaño de los mismos sea mayor del 25\%-30\% de la superficie articular.

El tratamiento quirúrgico puede hacerse en algunos casos de forma percutánea, o abierto si fuese preciso, utilizando la vía de abordaje de Wagner ${ }^{6}$. Durante el siglo XX se han utilizado multitud de técnicas distintas, dependiendo de las distintas épocas y de los materiales y medios existentes en cada momento (Tabla I).

La fractura de Rolando ${ }^{7}$ fue descrita en 1910 para fracturas en "Y" o en "T" de la base del $1^{\text {er }}$ metacarpiano. Hoy día se aplica a todas las fracturas intraarticulares conminutas de dicha zona.

El diagnostico se hace por radiología convencional, pudiendo usarse la TAC para valorar nú- mero y posición de los fragmentos. El tratamiento de las fracturas de Rolando siempre es quirúrgico, y dependerá del grado de conminución, pudiendo utilizarse agujas de Kirschner (con o sin injertos óseos), miniplacas de titanio, tracción de Lars Thoren $^{8}$ o similares, fijadores externos o técnicas combinadas (Figura 5). Para las secuelas se pueden practicar técnicas de salvamento como las artrodesis o artroplastias (Figura 6).

En el pulgar también son frecuentes las fracturas de las falanges, como la de la figura 7 donde se aprecia como el ligamento colateral cubital de la MCF ha arrancado un fragmento óseo que está volteado y que por tanto requiere su restitución y fijación quirúrgica.

En el tratamiento de las fracturas de los metacarpianos y las falanges, en general, para obtener un resultado óptimo aconsejamos hacer incisiones adecuadas, el manejo poco traumático de las partes blandas y la elección apropiada del método de osteosíntesis.

Creemos que una intervención correcta llevará al éxito siempre que se haga un seguimiento

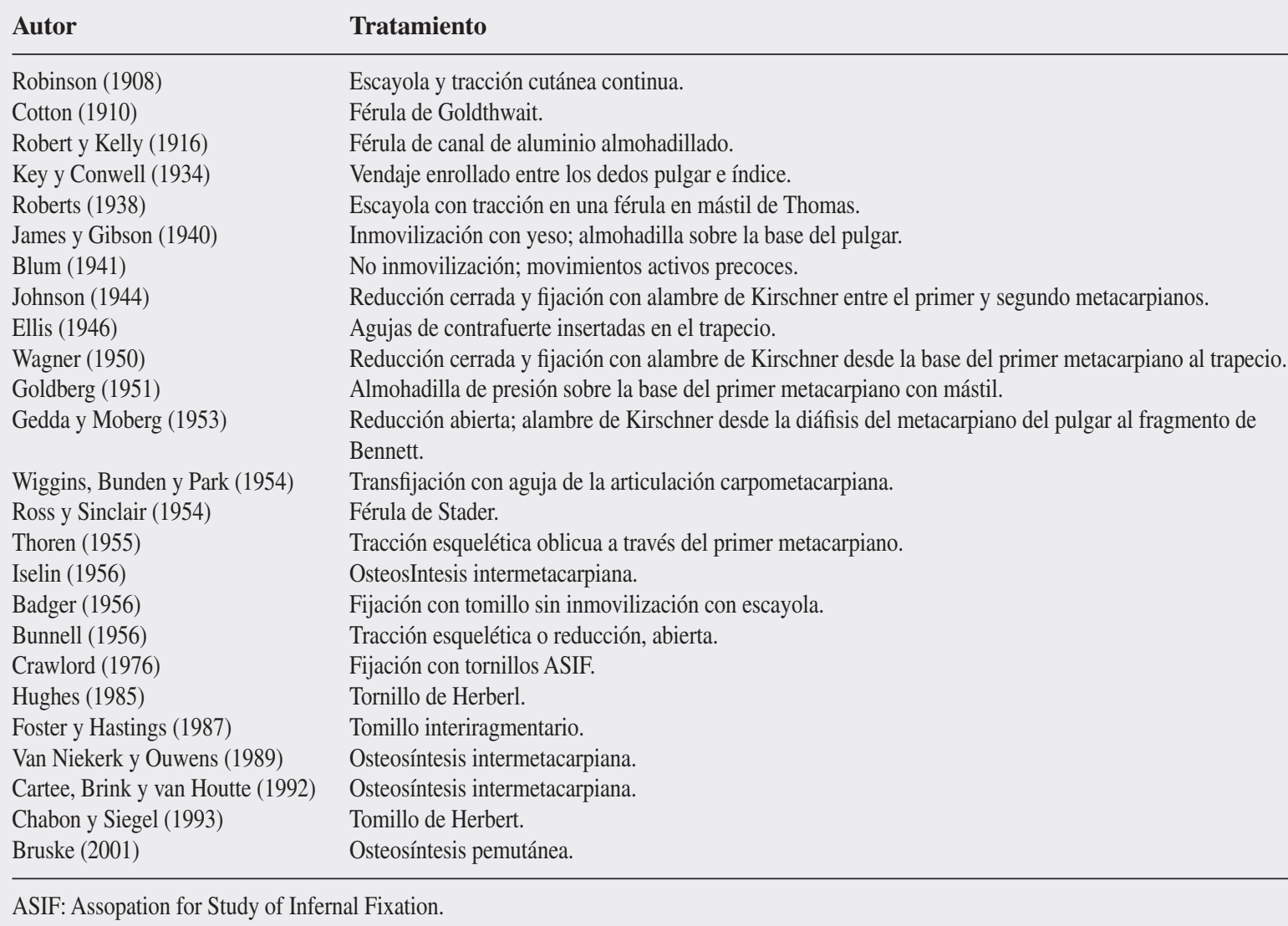

Tratamiento

Robinson (1908)

Cotton (1910)

Robert y Kelly (1916)

Key y Conwell (1934)

Roberts (1938)

James y Gibson (1940)

Blum (1941)

Johnson (1944)

Ellis (1946)

Wagner (1950)

Goldberg (1951)

Gedda y Moberg (1953)

Wiggins, Bunden y Park (1954)

Ross y Sinclair (1954)

Thoren (1955)

Iselin (1956)

Badger (1956)

Bunnell (1956)

Crawlord (1976)

Hughes (1985)

Foster y Hastings (1987)

Van Niekerk y Ouwens (1989)

Cartee, Brink y van Houtte (1992)

Chabon y Siegel (1993)

Bruske (2001)

Escayola y tracción cutánea continua.

Férula de Goldthwait.

Férula de canal de aluminio almohadillado.

Vendaje enrollado entre los dedos pulgar e índice.

Escayola con tracción en una férula en mástil de Thomas.

Inmovilización con yeso; almohadilla sobre la base del pulgar.

No inmovilización; movimientos activos precoces.

Reducción cerrada y fijación con alambre de Kirschner entre el primer y segundo metacarpianos.

Agujas de contrafuerte insertadas en el trapecio.

Reducción cerrada y fijación con alambre de Kirschner desde la base del primer metacarpiano al trapecio.

Almohadilla de presión sobre la base del primer metacarpiano con mástil.

Reducción abierta; alambre de Kirschner desde la diáfisis del metacarpiano del pulgar al fragmento de Bennett.

Transfijación con aguja de la articulación carpometacarpiana.

Férula de Stader.

Tracción esquelética oblicua a través del primer metacarpiano.

OsteosIntesis intermetacarpiana.

Fijación con tomillo sin inmovilización con escayola.

Tracción esquelética o reducción, abierta.

Fijación con tornillos ASIF.

Tornillo de Herberl.

Tomillo interiragmentario.

Osteosíntesis intermetacarpiana.

Osteosíntesis intermetacarpiana.

Tomillo de Herbert.

Osteosíntesis pemutánea.

ASIF: Assopation for Study of Infernal Fixation.

Tabla I: ASIF (Association for Study of Internal Fixation). Tomado de Stern ${ }^{2}$. 


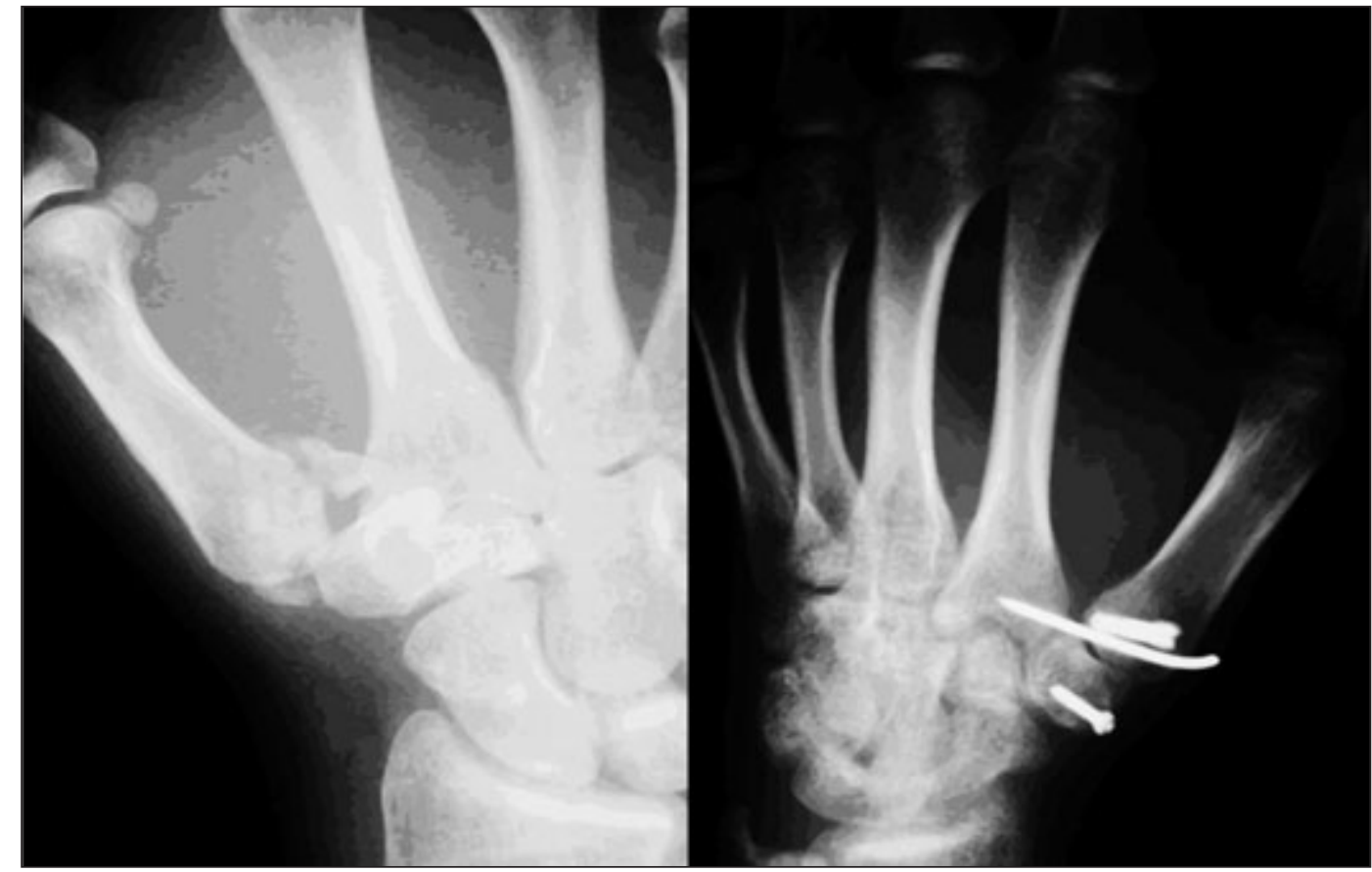

Figura 5: Fractura de Rolando. Osteosíntesis.

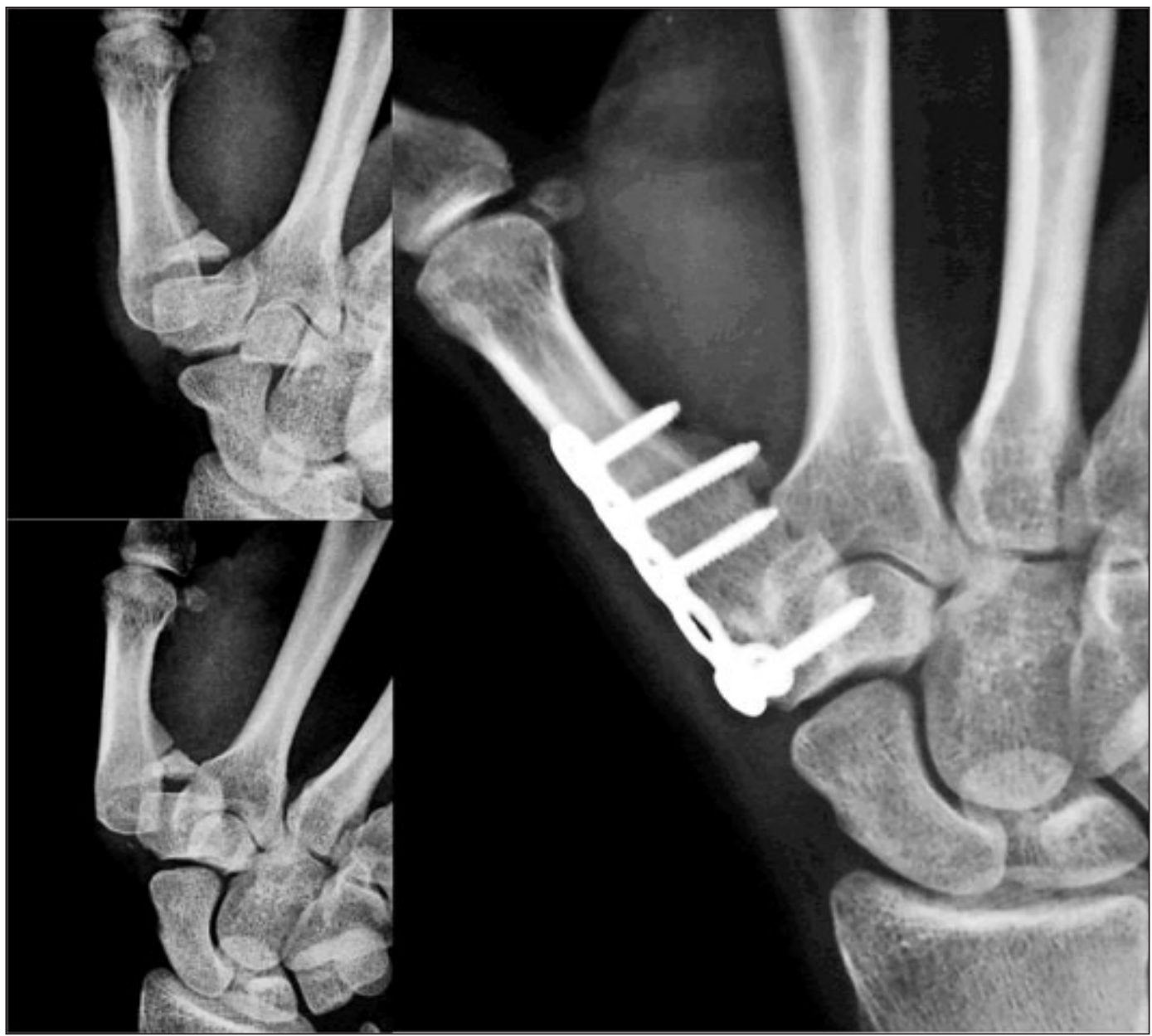

Figura 6: Artrodesis trapecio-metacarpiana por secuelas de fractura de Bennett. 


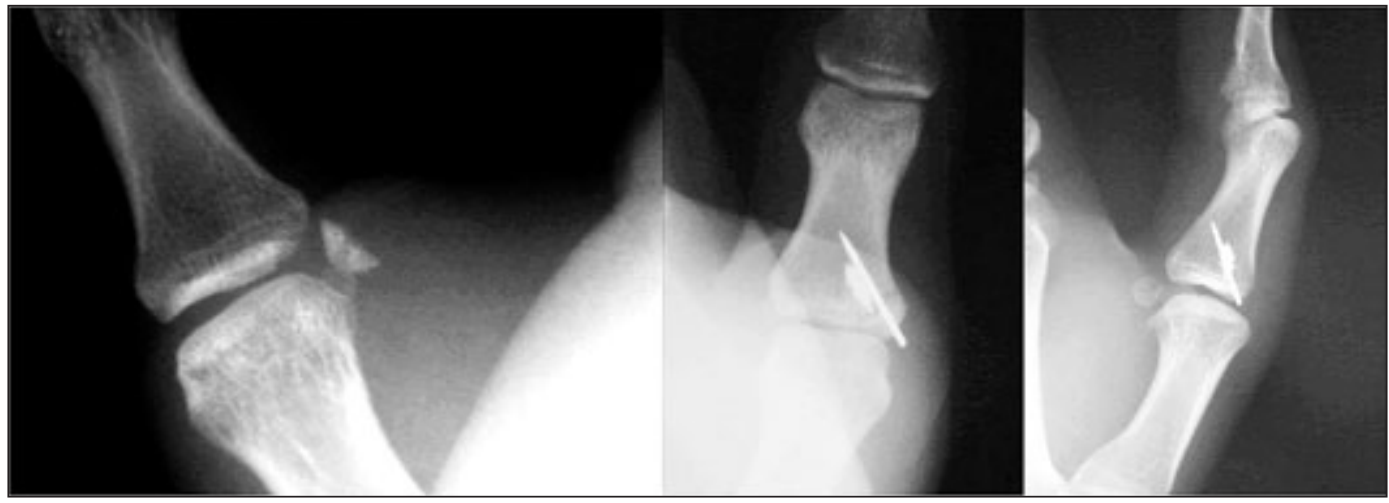

Figura 7: Fractura base F1 pulgar. Osteosíntesis.

preciso de la lesión y se practique una rehabilitación adecuada.

Debemos procurar no caer en la tentación de hacer indicaciones quirúrgicas innecesarias y recomendamos que la osteosíntesis sea realizada por cirujanos con preparación.

Capítulo especial requieren las denominadas fracturas complejas, que son ni más ni menos que importantes lesiones óseas que además se acompañan de la afectación de otros tejidos, como la piel, fascias, músculos, tendones, vasos o nervios. Suelen ser causadas por aplastamientos de la mano con rodillos, compresiones, avulsiones (lesiones por aplastamiento-tracción), o cizallamientos.
Existen unos principios para el manejo quirúrgico de estas lesiones complejas, como son el desbridamiento precoz, la resección de tejidos necróticos, el drenaje de hematomas, la descompresión de los compartimentos, la estabilización ósea, ya sea con agujas de Kirschner (Figura 8), placas o fijadores externos (Figura 9), y si fuese preciso la revascularización de la extremidad y las neurorrafias, con suturas o injertos. Con relativa frecuencia en ciertas lesiones complejas se precisa de distintos métodos de fijación y de actuación sobre partes blandas, incluida la cobertura cutánea con injertos, o colgajos libres o pediculados (Figura 10).

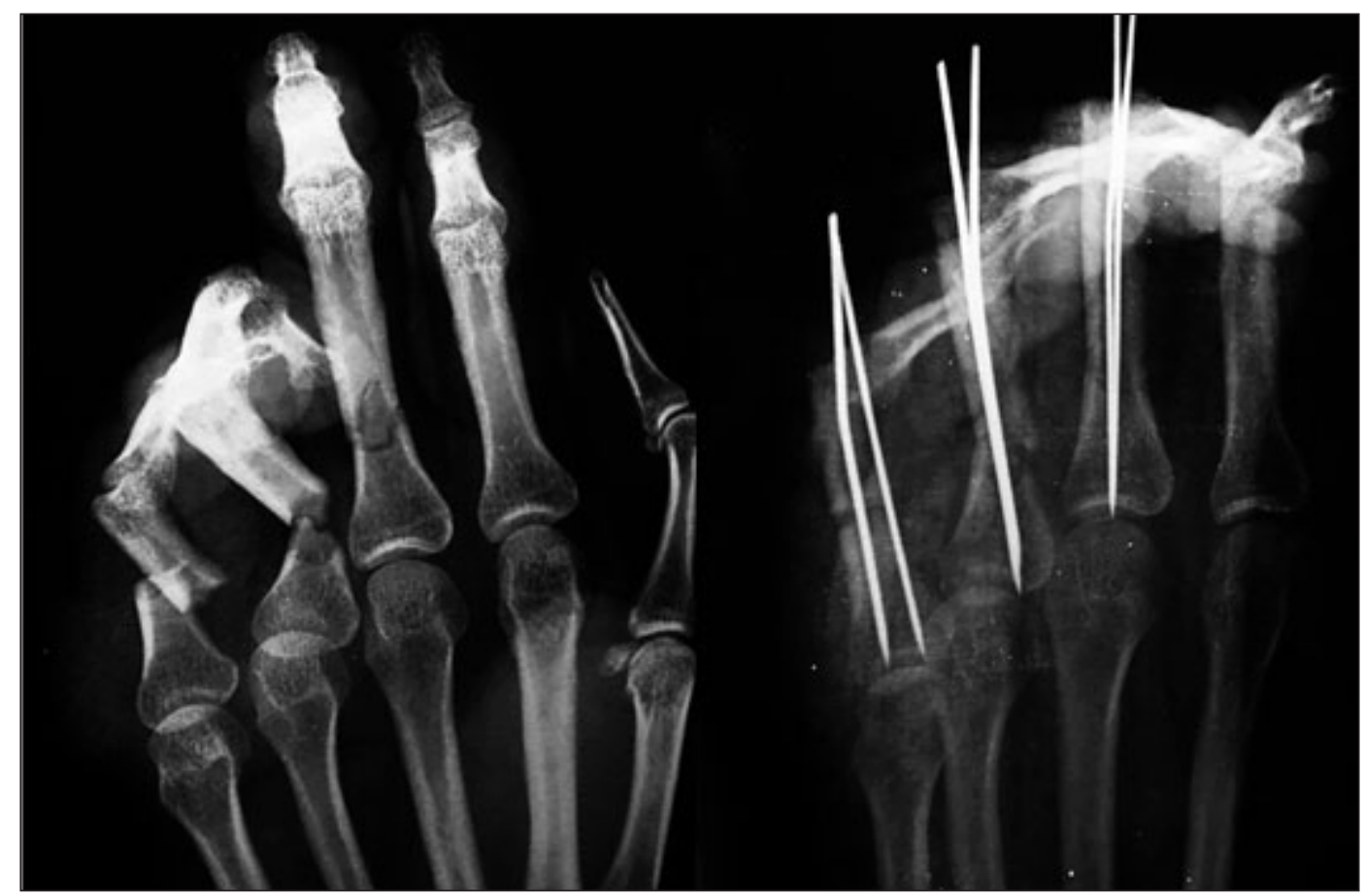

Figura 8: Lesión compleja de falanges. Estabilización con agujas de Kirschner. 


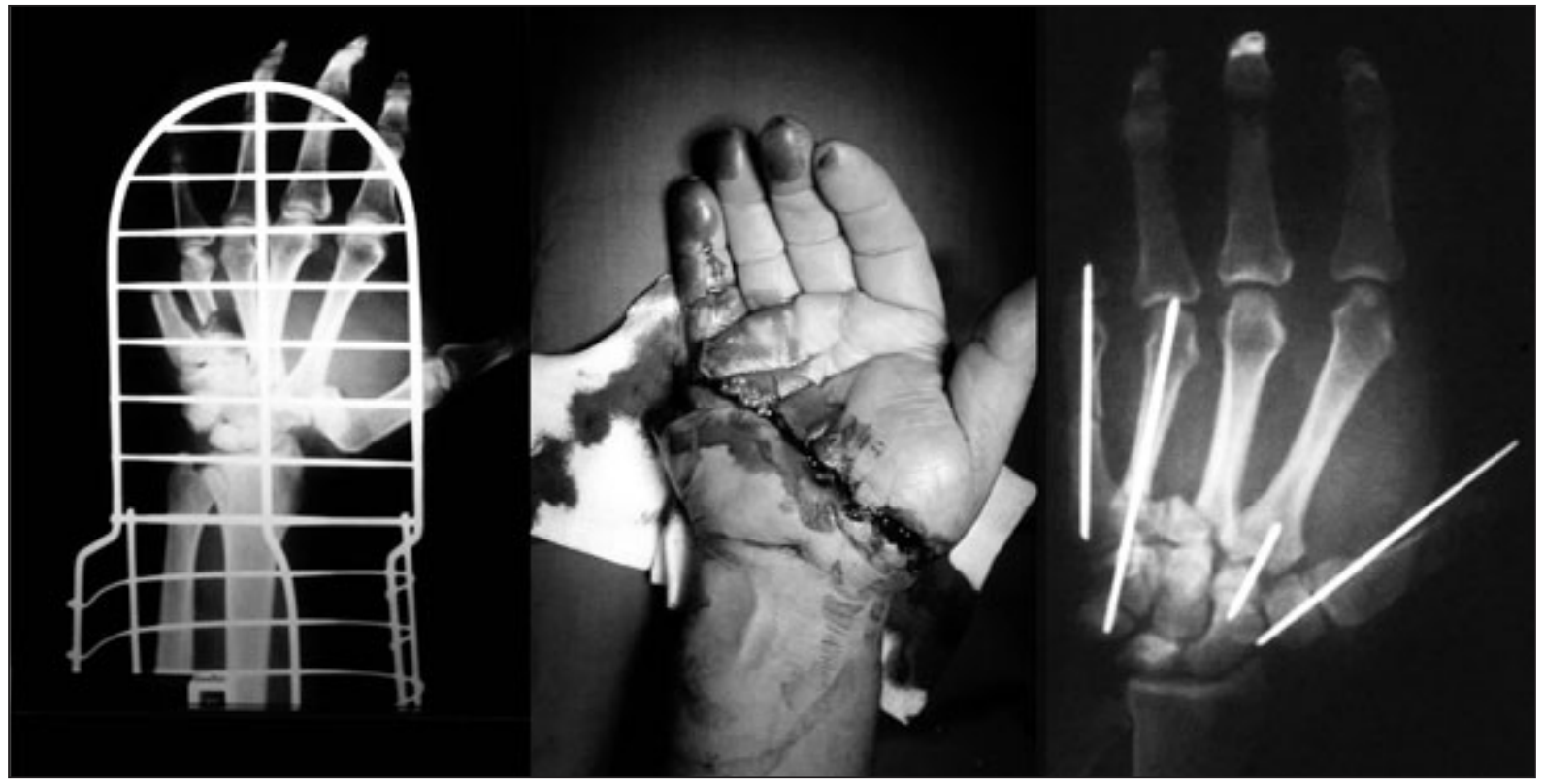

Figura 9: Lesión compleja de metacarpianos. Fijación temporal con agujas.

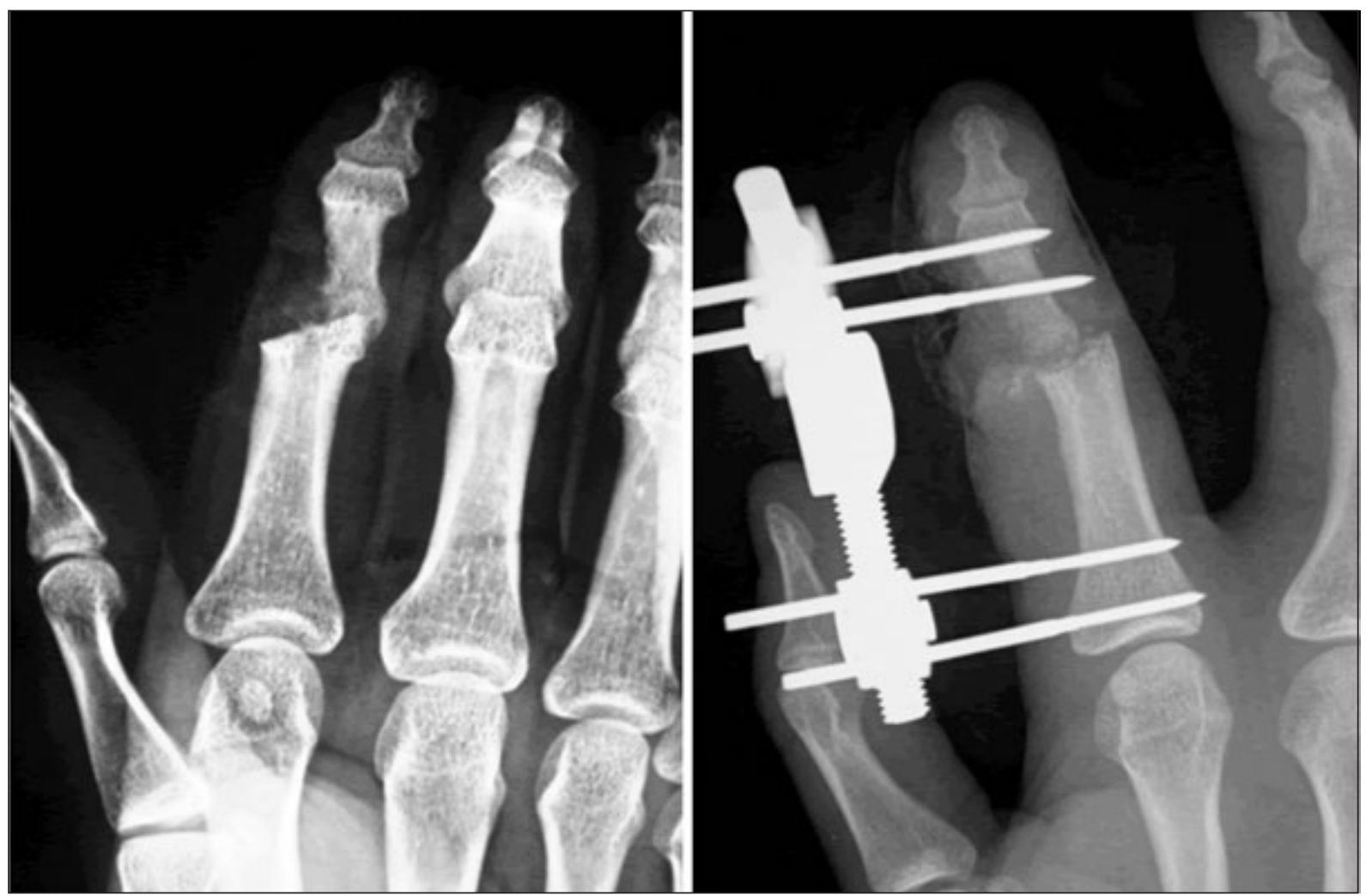

Figura 10: Fracturas abiertas con pérdida de sustancia. Fijación externa.

En las fracturas complejas de las manos el porcentaje de complicaciones es muy elevado, y así podemos encontrarnos con importantes rigideces articulares, necrosis cutáneas, seudoar- trosis y necrosis óseas, isquemias musculares y fibrosis, o infecciones.

Como conclusión en cuanto a las fracturas complejas de la mano podríamos decir que 


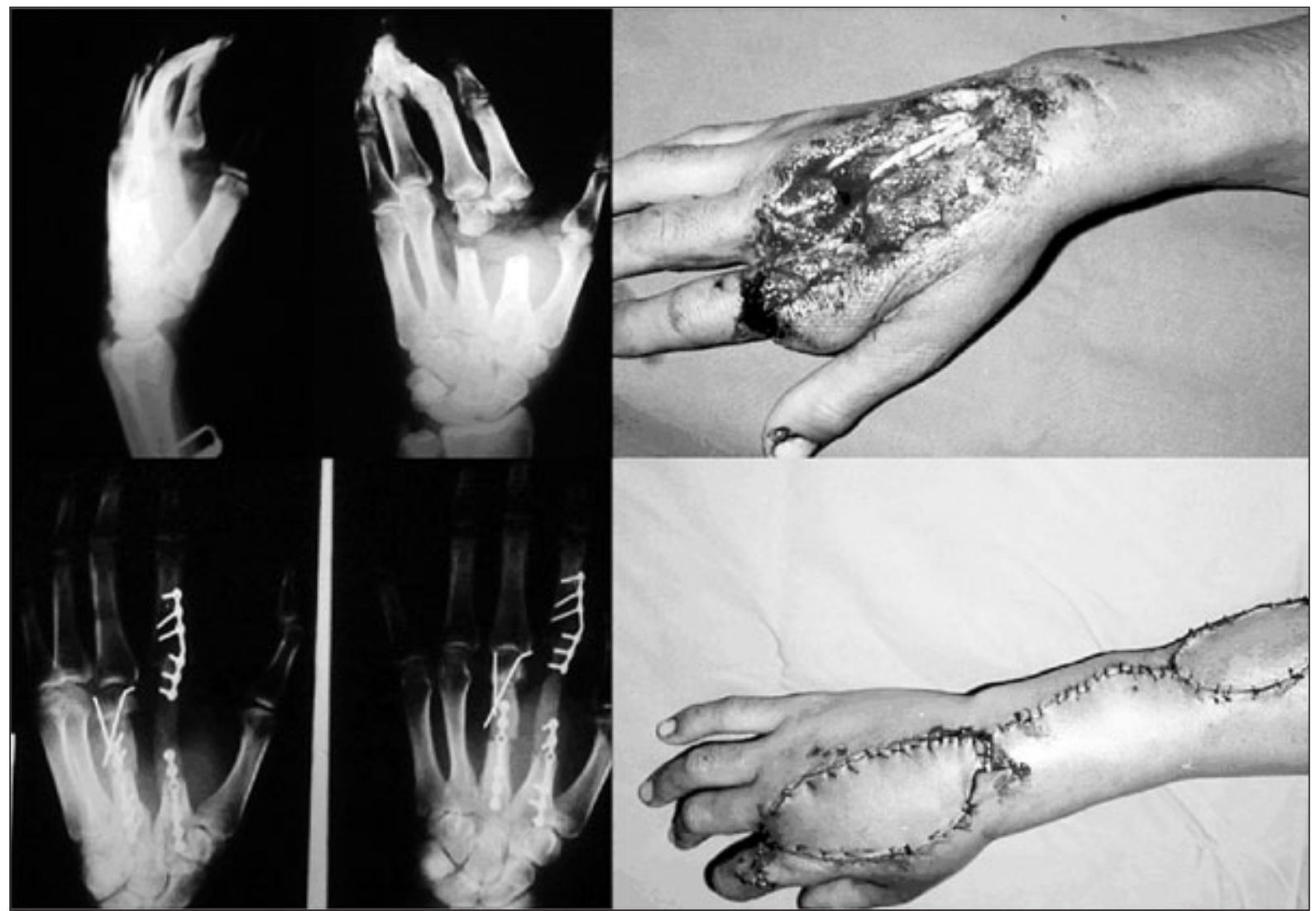

Figura 11: Lesión compleja de metacarpianos y falange. Osteosíntesis y cobertura cutánea.

Las fracturas complejas de metacarpianos y falanges son frecuentes en los aplastamientos de la mano.

- Las lesiones por aplastamiento casi siempre son graves.

- Hay lesiones cerradas que pueden esconder graves daños internos.
- Hay que procurar repararlo todo en un tiempo.

- La movilización debe ser inmediata, o lo más precoz posible.

- La rigidez en distintos grados será la norma.

- En las fracturas complejas de la mano abundan las complicaciones.

\section{BIBLIOGRAFÍA}

1. Swanson $A B$ : Fractures involving the digits of the hand. Orthop Clin North Am, 1970; 1: 261-74.

2. Stern PJ. Fracturas de los metacarpianos y falanges. En: Green DD, Pederson WC, Hotchkiss RN, et al. (Eds). Green's Cirugía de la Mano. Ed. en Español. vol 1. Madrid: Ed Marbán SL, 2007: 277-341.

3. Jupiter JB, Belsky MR. Fracture and dislocations of the hand.
En: Browner BD, Jupiter JB, Levine AM, et al. (Eds). Skeletal Trauma. Philadelphia: WB Saunders, 1992: 925-1024.

4. Wolfe SW, Elliott AJ. Metacarpal and carpometacarpal trauma. En: Peimer CA (Ed). Surgery of the Hand and Upper Extremity. New York: McGrawHill, 1996: 883-920.

5. Bennett EH. Fractures of the metacarpal bones. Dublin $\mathrm{J}$ Med Sci, 1882; 73: 72-5.
6. Wagner CJ. Methods of treatment of Bennett's fracturedislocation. Am J Surg, 1950; 80: 230-1.

7. Rolando S. Fracture de la base du premier metacarpien et principalment sur une variété non encore décrite. Presse Méd, 1910; 33: 303-4.

8. Thoren L. A new method of extension treatment in Bennett's fracture. Acta Chir Scand, 1956; 110: 485-93. 